\title{
Étude des cancers coliques du sujet jeune
}

Janick Selves, Sylviane Olschwang

$>$ Le cancer colorectal du sujet jeune (avant 45 ans) est une maladie souvent d'emblée agressive, de localisation plutôt distale avec fréquemment une faible différenciation tumorale. La majorité de ces tumeurs surviennent en dehors des syndromes héréditaires connus (syndrome de Lynch ou polypose). Une étude globale des anomalies génétiques somatiques de ces tumeurs a été initiée, soutenue par le programme Carte d'Identité des Tumeurs de la Ligue Nationale Contre le Cancer. Les résultats préliminaires de I'analyse montrent que les tumeurs développées avant 45 ans présentent un profil d'expression particulier. Une signature moléculaire de ces tumeurs du sujet jeune est en cours de validation. Leur profil génomique n'est en revanche pas différent de celui des cancers développés plus tardivement. <
Le cancer colorectal est caractérisé par une instabilité génétique, qui se traduit par au moins deux phénotypes des cellules tumorales [1]:

- L'instabilité génomique, visible particulièrement sur I'ADN microsatellite, dite MSI.

- L'instabilité chromosomique, avec des anomalies de nombre des chromosomes ou de segments chromosomiques, pertes, gains, qui se traduit à l'échelle moléculaire par des déséquilibres alléliques sans instabilité de I'ADN microsatellite, dite MSS.

Ces deux catégories ne sont pas équivalentes en proportion, le type MSI représentant seulement $8 \%$ à $15 \%$ de la totalité des cancers colorectaux. La plupart des cancers colorectaux survient de manière sporadique et à un âge tardif (médiane de survenue 74 ans); dans une petite proportion, ils se développent dans un contexte personnel ou familial évocateur d'une prédisposition génétique constitutionnelle, le syndrome de Lynch pour le type MSI et les polyposes pour le type MSS. Leur principale caractéristique commune dans ces derniers cas

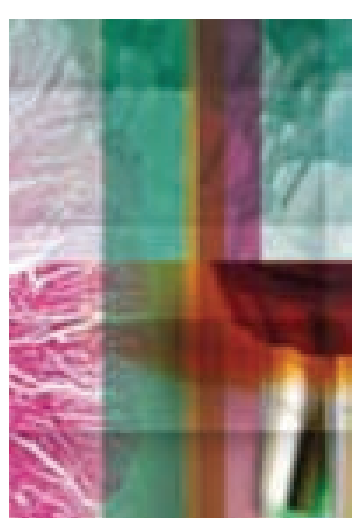

J. Selves : Inserm U563, CPTP, CHU Purpan, BP 3028, 31024 Toulouse Cedex 3, France. janick.selves@ toulouse.inserm. fr S. Olschwang : UMR 891, Département d’Oncogénétique, Centre de Recherche en Cancérologie de Marseille, Institut Paoli-Calmettes, 27, boulevard Leï Roure, 13009 Marseille, France. sylviane.olschwang@ inserm.fr

est un développement précoce, avec une moyenne d'âge au diagnostic inférieure à 50 ans (Figure 1). Le mécanisme des tumeurs MSI est bien élucidé et les gènes MMR en sont responsables. Dans les tumeurs MSS, les mécanismes de tumorigenèse ne sont pas tous identifiés. En particulier, une polypose n'est pas systématiquement présente en cas de développement précoce, il s'agit même d'une situation rare. Plusieurs études ont été menées pour tenter d'identifier un nouveau mécanisme qui permettrait d'expliquer ces situations, mais elles se sont intéressées à des patients sélectionnés diversement pour l'âge au diagnostic, allant de 35 à 50 ans, sans qu'une limite ne puisse être choisie autrement que de manière empirique [2, 3]. Le choix qu'a fait l'équipe toulousaine, compte tenu de ces incertitudes, a été de placer la limite à 45 ans.

Les cancers diagnostiqués avant 45 ans, hors polypose, ont une présentation un peu particulière, de localisation en majorité distale, voire rectale, sont de faible différenciation avec une composante mucineuse fréquente, et de stade histologique le plus souvent avancé, avec envahissement ganglionnaire ou viscéral. Ce sont donc des maladies d'emblée agressives $[4,5]$. De plus, il y a relativement peu de présentations familiales (30\% des cas), mais de fréquentes localisations multiples, métachrones ou synchrones. 


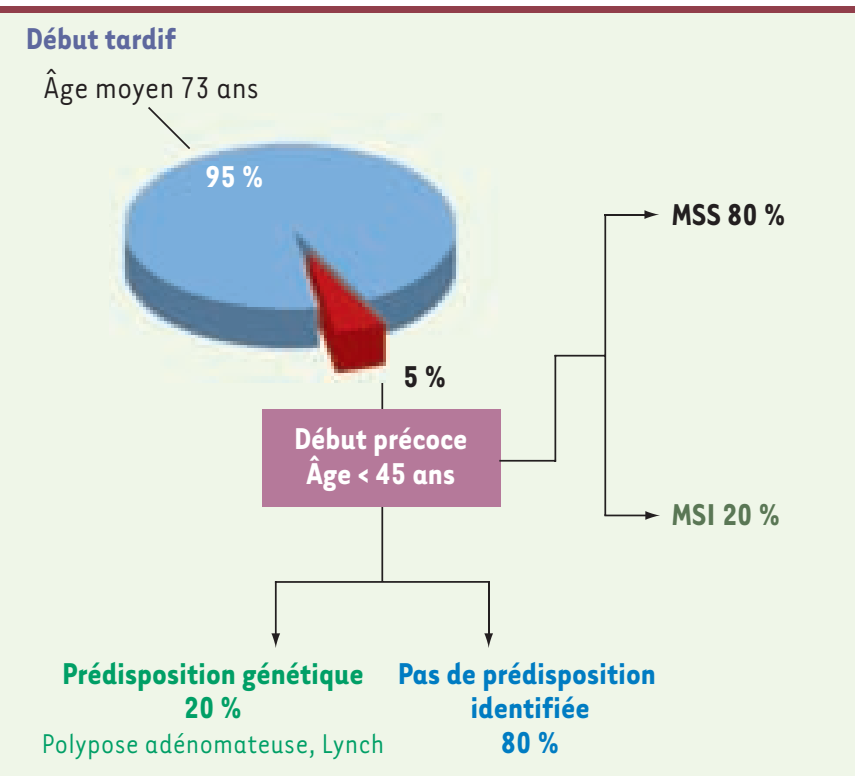

Figure 1. Caractéristiques générales des cancers colorectaux en fonction de l'âge au diagnostic.

\section{Analyse du transcriptome}

À Toulouse, le travail de sélection des patients atteints d'un cancer colorectal à un âge jeune a été possible grâce à la mise en place d'une recherche systématique de syndrome de Lynch, soutenue par un PHRC il y a quelques années. Ce travail coordonné par le Pr Rosine Guimbaud, oncologue digestif et oncogénéticienne, a permis à l'équipe de recenser plus de 200 patients de moins de 50 ans sans prédisposition héréditaire identifiée avec détermination du statut microsatellitaire (MSI ou MSS).

L'étude globale des anomalies génétiques acquises dans les tumeurs de ces patients est soutenue par le programme "Carte d'Identité des Tumeurs » de la LNCC. II a été convenu, outre l'étude de ces tumeurs, de comparer leurs profils aux tumeurs développées dans un contexte sporadique très probable, donc diagnostiquées après 60 ans. Les différentes sélections ultérieures, sur la disponibilité et la qualité du matériel biologique, ont permis l'analyse complète du génome et du transcriptome de 48 tumeurs de patients de moins de 45 ans et 50 de plus de 60 ans. Pour les analyses de transcriptome, seules 29 tumeurs chez des patients jeunes ont pu être étudiées; 25 avaient une tumeur de type MSS et 4 de type MSI.

Bien que l'étude soit en cours actuellement, quelques résultats préliminaires peuvent être discutés. Ils concernent la caractérisation du transcriptome, avec la recherche de sous-groupes et d'un lien avec les variables cliniques et biologiques, en particulier l'âge au diagnostic. Des analyses supervisées sur le profil MSI ou MSS et sur l'âge dans les tumeurs MSS ont également été menées.

$\varepsilon n$ résumé, le sexe et le statut MSI/MSS sont les paramètres les plus discriminants pour le profil d'expression. Des paramètres histologiques ou génétiques, non indépendants du profil MSI, apparaissent également discriminants tels que le caractère colloïde, l'existence d'un syndrome de Lynch, la perte d'expression d'une protéine MMR en immunohistochimie.

Au total, les profils d'expression définissent plusieurs sous-groupes de tumeurs en fonction de leur statut génomique (MSI ou MSS) et de l'âge de survenue de la tumeur.

L'analyse supervisée sur l'âge définit une signature de 466 gènes regroupés en 5 catégories selon les variations d'expression, dont la validation est en cours. L'analyse des différents groupes de gènes fait apparaître que les gènes dérégulés dans les tumeurs des patients jeunes concernent le métabolisme cellulaire, l'apoptose et la modulation de la réponse immunitaire, l'adhésion cellulaire et la migration, ainsi que la prolifération cellulaire.

La suite de l'étude va consister à essayer de mettre en relation le profil d'expression et les altérations génomiques globales ou les mutations de gènes cibles, comme KRAS, EGFR, BRAF par exemple. II est également important de restreindre la liste des gènes discriminants à un nombre compatible avec une caractérisation génomique et fonctionnelle. Pour confirmer ces observations, il faudra mobiliser d'autres structures de soins pour augmenter l'effectif des tumeurs diagnostiquées avant 45 ans, à travers le cancéropôle qui nous fédère, à savoir la région Grand-Sud-Ouest.

\section{Profil génomique}

Le matériel d'étude sélectionné pour l'analyse génomique à Marseille était une série de cancers colorectaux diagnostiqués avant l'âge de 50 ans, dont

\section{Données démographiques}

Hommes : $41 \quad$ Femmes : 28

Moyenne d'âge au diagnostic : 45 ans $\langle 29-50\rangle$

Localisation tumorale

Côlon droit : 22

Côlon gauche : 29

Rectum : 18

\section{Stade histologique}

$1: 6$

II : 27

III $: 36$

Tableau I. Résumé des données démographiques et des paramètres cliniques et histologiques des tumeurs. 
le contexte de survenue n'évoquait aucune forme connue de susceptibilité au cancer:

- maladies inflammatoires chroniques de l'intestin connues;

- prédispositions génétiques majeures pour polypose typique connue ou atypique par analyse systématique du gène $M Y H$; - profil MSI évocateur de l'existence d'un syndrome de Lynch.

Les patients avec tumeur sous-douglassienne ayant été traités par radiothérapie avant intervention chirurgicale ont également été exclus.

Après contrôle de la qualité du prélèvement (infiltration de la tumeur par des cellules normales $40 \%$ ) et de l'ADN, une série de 69 échantillons (Tableau I) ont été hybridés selon la technique Agilent sur des puces de 244000 oligonucléotides et les données brutes ont été traitées avec le logiciel CGH Analytix.

L'analyse globale des pertes chromosomiques retrouve, sans différence avec le profil des tumeurs de diagnostic à un âge plus tardif, une très forte fréquence de perte du $17 p$ et du 18 , puis du $5 q$ et du $8 p$, et une forte fréquence de gain du $8 q$, du $13 q$ et du $20 q$.

Ces données sont obtenues après analyse globale des 244000 points de mesure, impossibles à utiliser à la recherche d'association pour plusieurs raisons: la proximité physique des points sur un même bras chromosomique fait que des événements proches ne surviennent pas ensemble par hasard. Les recherches d'associations intra-chromosomiques ne sont donc pas informatives. D'autre part la multiplicité des comparaisons 2 à 2 ne permettrait pas d'envisager une interprétation au sens statistique. En revanche, la simi-

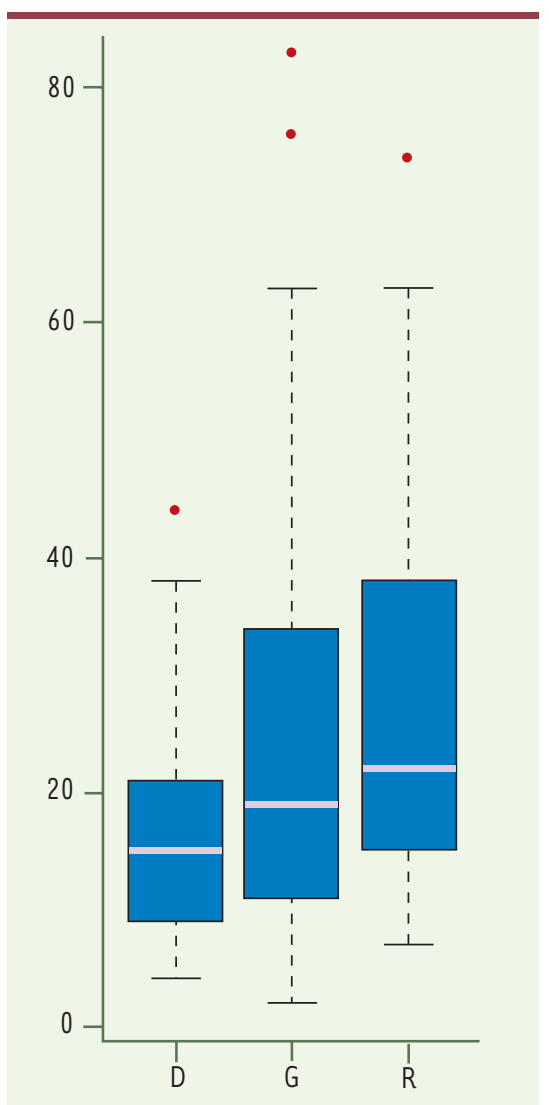

litude des informations fournies par la mesure des points physiquement proches permet de procéder à de comparaisons par segments chromosomiques regroupant des informations concordantes. L'étape ultérieure de l'analyse a donc consisté à utiliser des programmes de segmentation élaborés sur des modèles cellulaires assez purs et à les valider sur le jeu de données expérimentales, sachant que l'intensité des signaux obtenus à chaque point diminue d'autant que le prélèvement tissulaire contient

Figure 2. Localisation colorectale des tumeurs en fonction du nombre de cassures chromosomiques. une forte proportion de cellules non tumorales, et que la différence de signal de deux segments adjacents peut de ce fait être assez petite. À l'issue de cette étape, les tumeurs ont donc été définies par des points de cassure, matérialisant des différences d'intensité de fluorescence de deux segments donc des statuts différents (perte, gain...). Un total de 1675 segments indépendants, représentant les 22 autosomes, a été défini à partir des 69 tumeurs.

Une première représentation des altérations génomiques, faisant état des pertes et des gains de segments, a confirmé les observations initiales. Les segments chromosomiques les plus fréquemment perdus comprenaient les gènes dont l'implication à différentes étapes de la tumorigenèse colorectale est connue, $p 53$ sur le $17 p$, SMAD4 et DCC sur le $18 q$ et $A P C$ sur le $5 q$. D'autres segments chromosomiques, moins fréquemment perdus, permettaient également de retrouver des gènes connus, FHIT, CDKN2A, PTEN par exemple.

Une autre représentation consiste à matérialiser la densité de points de cassure observée sur une unité de longueur, par exemple $10^{6}$ nucléotides. II apparaît que certaines régions sont très riches en points de cassure, comme une portion de $10^{7}$ nucléotides du $8 p$ qui en contient 82 , alors que le chromosome 2 entier en contient 38. II existe donc une distorsion importante entre la densité de points de cassures et la taille des chromosomes.

Des corrélations de ces deux représentations à l'échelle physique, en y intégrant la carte des CNV (Copy Number Variation), et des recherches d'associations inter-chromosomiques sont en cours. Dans la mesure où aucune différence importante dans la fréquence, le type et la localisation des altérations chromosomiques n'était observée entre les tumeurs diagnostiquées avant et après 50 ans, nous avons procédé à une comparaison interne du profil génomique des tumeurs diagnostiquées avant 45 ans et entre 45 et 50 ans. Là encore, il n'y a aucune particularité, et le choix de la limite d'âge reste donc empirique.

Par ailleurs, nous avons comparé les profils génomiques avec le degré d'instabilité des tumeurs, en considérant non pas la proportion globale de génome altéré, mais le nombre de cassures chromosomiques. Si l'on considère, sur le plan mécanique, qu'une altération d'un grand chromosome est souvent partielle, c'est-à-dire qu'elle résulte volontiers d'une délétion ou duplication localisée, le bras chromosomique correspondant présentera deux points de cassure bordant la région altérée, ou un lorsque cette altération 
est télomérique. À l'inverse, les chromosomes acrocentriques et/ou de petite taille sont plus souvent le siège d'anomalies touchant un bras ou un chromosome dans son intégralité. Les tumeurs ont été classées en fonction du nombre de cassures, dans deux groupes, la distribution des valeurs (extrêmes 2-80) présentant une inflexion à la valeur 30 , selon qu'elles présentaient plus ou moins de 30 cassures. Le profil génomique de ces deux groupes est comparable. En revanche, les tumeurs présentant plus de 30 cassures sont plus volontiers localisées dans le côlon gauche et le rectum, sans signification statistique (Figure 2).

\section{Conclusion}

En résumé, l'analyse globale des profils génomiques ne détecte aucune caractéristique, qui pourrait traduire l'existence d'un sous-groupe particulier de tumeurs. Les cancers colorectaux de type MSS développés avant 50 ans, après élimination des prédispositions actuellement connues, inflammatoires et génétiques, sont homogènes et ne sont pas différents des cancer colorectaux sporadiques MSS plus tardifs. À côté des anomalies connues de quelques gènes, nous envisageons de poursuivre l'analyse par une recherche d'associations d'autres anomalies, afin d'identifier dans les voies connues comme p53, TGF, Wnt, RAS ou d'autres, des mécanismes alternatifs ou complémentaires de ceux déjà décrits. $\diamond$

\section{SUMMARY}

Early-onset colorectal carcinoma study

Early-onset colorectal carcinoma (before the age of 45 years) are a distinct clinical group characterized by frequent distal location, poor cellular differentiation and aggressive evolution. A systematic study of early-onset cancers without familial predisposition in the context of the CIT program of "la Ligue Nationale Contre le Cancer" has been set up to characterize molecular mechanisms of carcinogenesis of these tumors. Preliminary results of transcriptome analysis show different gene-expression profiles among age. The next step will consist in validating a molecular signature specific for early-onset carcinoma. Genomic profile does not differ from that observed in late-onset colon cancers. $\diamond$

\section{RÉFÉRENCES}

1. Grady WM, Carethers JM. Genomic and epigenetic instability in colorectal cancer pathogenesis. Gastroenterology. 2008, 3 septembre online.

2. Mourra N, Zeitoun G, Buecher B, et al. High frequency of chromosome 14 deletion in early-onset colon cancer. Dis Colon Rectum 2007 ; 11 : 1881-6.

3. Boardman LA, Johnson RA, Peterson GM, et al. Higher frequency of diploidy in young-onset microsatellite-stable colorectal cancer. Clin Cancer Res $2007 ; 15: 2323-8$.

4. Mitry $\varepsilon$, Benhamiche AM, Jouve JL, et al. Colorectal adenocarcinoma in patients under 45 years of age: comparison with older patients in a welldefined French population. Dis Colon Rectum $2001 ; 44: 380-7$.

5. O'Connell JB, Maggard MA, Liu JH, et al. Rates of colon and rectal cancers are increasing in young adults. Am Surg 2003; 69: 866-72.

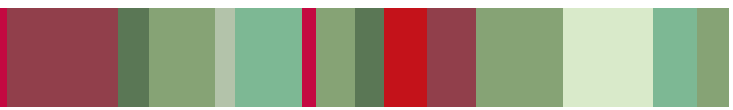

TIRÉS À PART

S. Olschwang 